Journal of Experimental and Clinical Medicine https://dergipark.org.tr/omujecm

Research Article

J Exp Clin Med

2021; 38(3): 216-220

doi: $10.52142 /$ omujecm.38.3.2

\title{
Reverse shoulder arthroplasty-early results
}

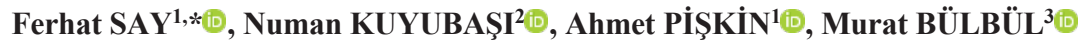

\begin{abstract}
${ }^{1}$ Department of Orthopaedics and Traumatology, Faculty of Medicine, Ondokuz Mayıs University, Samsun, Turkey
${ }^{2}$ Department of Orthopaedics and Traumatology, Faculty of Medicine, Kütahya Sağlık Bilimleri University, Kütahya, Turkey

${ }^{3}$ Department of Orthopaedics and Traumatology, Faculty of Medicine, Medipol University, İstanbul, Turkey
\end{abstract}

\begin{abstract}
\begin{tabular}{cccc}
\hline Received: $20.05 .2020 \quad \bullet \quad$ Accepted/Published Online: 09.02.2021 & $\bullet$ & Final Version: 23.04 .2021 \\
\hline
\end{tabular}
\section{Abstract}

Although reverse shoulder arthroplasty is used in massive rotator cuff tears, it is also used in proximal humerus fractures and shoulder arthroplasty revision. In this study, we aimed to examine the early radiological and clinical results of patients undergoing reverse shoulder arthroplasty with different diagnoses. Between 2010 and 2013, reverse shoulder arthroplasty was applied to 10 patients (4 men, 6 women) with the diagnosis of arthropathy due to rotator cuff tear (n: 7), multi-fragmentary proximal humerus fracture (n: 2) and hemiarthroplasty revision (n: 1). The median age of the patients was 74 (64-85) years and the median follow-up was 15.5 (3-35) months. Patients were assessed in terms of joint range of motion, Constant score and Visual Pain Scale (VAS) and radiological examinations before and after surgery. Preoperative active shoulder flexion, abduction, internal and external rotation degrees of the patients were 65, 30, 40 and 50 degrees, and 105, 95, 30, 57.5 degrees respectively in their final controls. Constant score was 20.5 (14-63) preoperatively, and 54.5 (38-64) in the final controls ( $<<0.05)$. While the preoperative VAS was 7 (3-9), it was found to be $1.5(1-3)$ in the final controls $(\mathrm{p}<0.05)$. As a complication, scapular notching was observed in one patient. Internal external rotation values were better in the rotator cuff arthropathy group than the fracture group. Reverse shoulder arthroplasty, especially in the treatment of patients with rotator cuff arthropathy, has good early results and a low complication rate. We recommend paying attention to the glenoid component location to avoid scapular notching.
\end{abstract}

Keywords: proximal humerus fracture, rotator cuff arthropathy, scapular notching, reverse shoulder arthroplasty

\section{Introduction}

Reverse shoulder arthroplasty (RSA), first designed by Paul Grammont in France in the 1980s, extends the moment arm of the deltoid muscle by shifting the shoulder rotation center medially and inferiorly. Thus, active forward flexion and abduction movement of the shoulder is provided with deltoid muscle strength (Grammont and Baulot, 1993). It has a more stable prosthesis design than total and partial shoulder prostheses (Matsen et al., 2007). Although RSA are used in massive rotator cuff tear arthropathy, it has also been found to be used in failed shoulder arthroplasty or internal fixation revisions, shoulder involvement of rheumatological diseases, tumor reconstruction, multi-fragmentary proximal humerus fractures (Boileau et al., 2006; Guery et al., 2006; Matsen et al., 2007; Levy and Badman, 2011; Melis et al., 2012; Shafritz and Flieger, 2012; Schwarz et al., 2021). In this study, we aimed to report the clinical and radiological results of RSA that have been performed with different diagnoses.

\section{Materials and Methods}

Between 2010 and 2013, a total of 10 patients, four males and six females, underwent shoulder replacement surgeries with RSA. The diagnoses were arthropathy due to massive rotator cuff tear in seven patients, multi-fragmentary proximal humerus fracture in two patients, and hemiarthroplasty revision in one patient. The indications for surgical treatment in patients diagnosed with arthropathy due to massive rotator cuff tear were despite conservative treatment for at least six months to continue the complaint, determining irreparable massive rotator cuff tears in magnetic resonance imaging (MRI) and accompanying pseudo-paralytic shoulder to tears. The pseudoparalytic shoulder was defined as anterior flexion less than 60 degrees accompanying anterior and superior instability (Shafritz and Flieger, 2012). Preoperative shoulder range of motion was measured with the help of a goniometer. In the preoperative evaluation, each patient underwent standard anterior-posterior and axillary shoulder radiography, MRI in addition to patients with rotator cuff arthropathy, and computed tomography (CT) examinations in addition to those with a proximal humeral fracture. The right shoulder of five patients and the left shoulder of five patients were operated.

In the surgical technique, the deltopectoral approach was used in all patients and the tenotomy was applied to the subscapularis tendon. The midpoint of the glenoid was found, 
and a Kirshner wire was sent from the inferior of this point with 15 degrees inferior, parallel to the scapular body. The glenoid was prepared by removing only superficial subchondral bone using a reamer. The baseplate was fixed to the scapula with at least two screws without using cement. Humerus diaphysis was reamed with suitable reamers and metaphysis was prepared with rasps. The humeral stem was placed with cement in an appropriate degree at 10 degrees retroversion. The glenoid sphere was fixed to the baseplate with a screw. A polyethylene insert of appropriate size was placed after the trials in the humeral system. The joint was reduced, and stability control was checked. In patients operated with the diagnosis of proximal humerus fracture, tuberculum majus, and tuberculum minus sutured anatomically with the help of the sutures (Fig. 1).

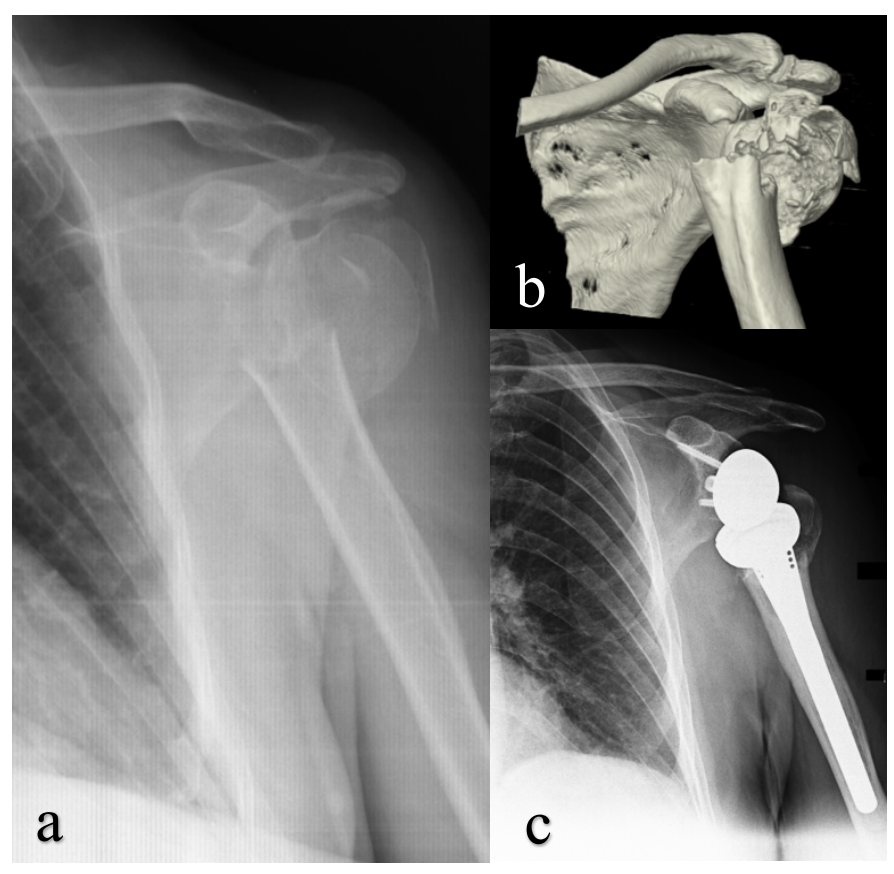

Fig. 1. Pre-operative anterior posterior shoulder radiography (a) threedimensional reconstruction computerized tomography image (b) and postoperative $4^{\text {th }}$ month control anterior posterior radiography (c) with the diagnosis of multi-fragmentary proximal humeral fracture

Shoulder arm sling was applied in the first six weeks after the operation and passive joint range of motion exercises were started in the early period. Active exercises were started after the sixth week, and muscle-strengthening exercises were performed after 12 weeks.

Patients were evaluated with joint range of motion, Constant and visual pain scale (VAS) scores and direct radiography, in their last controls. Constant score includes pain, degree of activity, and shoulder movements. The median age of the patients was 74 (64-85) years and the median followup was 15.5 (3-35) months.

The results are stated as median (minimum-maximum). SPSS for Windows v.16.0 (SPSS Inc., Chicago, IL, USA) program was used to evaluate the data. Wilcoxon test was used for statistical evaluation of preoperative and postoperative data. Statistical significance level was accepted as $\mathrm{p}<0.05$.

\section{Results}

Preoperative active shoulder flexion, abduction, internal and external rotation degrees were 65, 30, 40 and 50 degrees, respectively, post-operatively they were measured as 105, 95, 30, 57.5 degrees, respectively (Fig. 2, Tables 1, 2). Constant score was 20.5 (14-63) preoperatively, and 54.5 (38-64) in the final controls $(p<0.05)$. While the preoperative VAS was 7 (3$9)$, it was found to be $1.5(1-3)$ in the final controls $(p<0.05)$ (Table 2). As complications, none of the patients had hematoma, infection, instability, acromial insufficiency, glenoid and humeral component loosening. Scapular notching was observed in one patient. In this patient, radiologically notching had not affected the lateral edge of the scapula and reached the lower screw (Fig. 3). Scapular notching was classified as grade 1 according to the Sirveaux classification (Sirveaux et al., 2004). In this patient who was followed up conservatively with physical therapy, it was found that the range of motion was not affected despite the scapular notching.

\section{Discussion}

RSA is not a very new concept and was found as an alternative to other shoulder prostheses in the 1970s. However, due to the lateralization of the shoulder joint rotation center in early designs, the load on the glenoid increased, and early loosening occurred (Ramirez et al., 2012). In 1987, Paul Grammont created a new prosthesis concept, and in his biomechanical studies, it was shown that medialization of the rotation center by $10 \mathrm{~mm}$ would increase the deltoid abduction moment by $20 \%$, and the rotation center by $10 \mathrm{~mm}$ inferior would increase the deltoid abduction moment by 30\% (Baulot et al., 2011; Ramirez et al., 2012). Although the main indication of the reverse shoulder prosthesis is massive rotator cuff tear arthropathy, its indications have widened over time, it has found use in failed shoulder arthroplasty or internal fixation revisions, shoulder involvement of rheumatological diseases, tumor reconstructions, multi-fragmentary proximal humerus fractures (Boileau et al., 2006; Guery et al., 2006; Matsen et al., 2007; Levy and Badman, 2011; Melis et al., 2012; Shafritz and Flieger, 2012 Schwarz et al., 2021).

In our study, the etiologic diagnosis of patients was rotator cuff tear arthropathy, multi-fragmentary proximal humerus fracture, and hemiarthroplasty revision. When the results of our study were evaluated, shoulder abduction and flexion increased in the rotator cuff tear group patients. It was observed that there was no significant change in internal and external rotation values in patients operated with a diagnosis of fracture and revision. Although the number of patients was low for statistical comparison, we found the best functional outcome in the rotator cuff tear group. The functional outcome of RSA for the rotator cuff tear arthropathy has been reported better than revision arthroplasty and posttraumatic arthritis (Boileau et al., 2006; Wall et al., 2007). However, some authors reported similar functional outcomes of RSA for rotator cuff tear 
arthropathy and proximal humerus fracture with lower satisfaction in patients with an acute fracture (Sebastia-Forcada et al., 2020). The determinant of internal and external rotation in RSA applied in fracture etiology depends on the anatomical restoration and healing of tuberculum majus and minus (Levy and Badman, 2011; Gunst et al., 2021).

Table 1. Demographic information and study data of patients

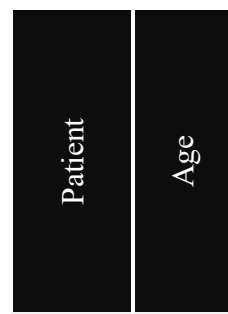

1

\begin{tabular}{l|l}
12 & 73 \\
\hline
\end{tabular}

\begin{tabular}{l|l}
3 & 83
\end{tabular}

\begin{tabular}{l|l}
4 & 80
\end{tabular}

55

64

\begin{tabular}{l}
68 \\
\hline
\end{tabular}

$8 \quad 78$

92

10

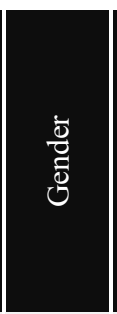

F

M

F

M

F

F

CTA

M HR

F CTA

F

M

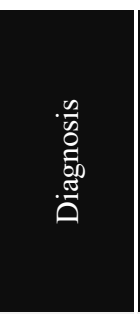

CTA

CTA 8

CTA 10

PHF 6

PHF 3

A 31

TA 25

CTA 35

CTA 34

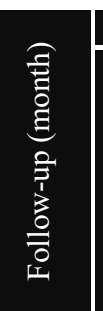

$11 \quad 45$

50

150

-

$-$

40

70

80

60

70

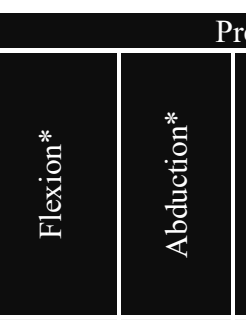

20

30

140

$-$

$-$

30

60

20

30
Preoperative

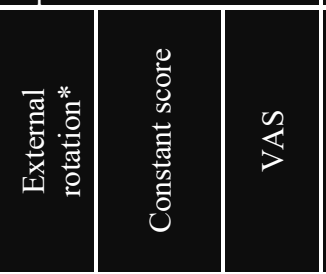

60

50

70

55

20

40

40
14

\begin{tabular}{l|l}
27 & 7
\end{tabular}

\begin{tabular}{l|l}
27 & 7 \\
\hline
\end{tabular}

$-$

16

19

22

17

27

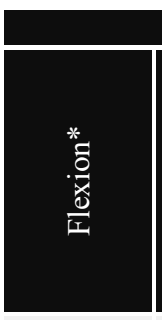

170

165

70

90

80

145

100

130

100

110

Last control

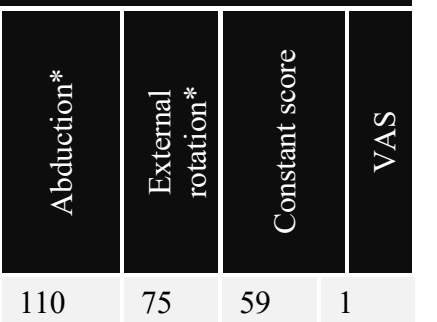

\begin{tabular}{l|l|l|l}
100 & 80 & 64 & 1
\end{tabular}

\begin{tabular}{l|l|l|l}
45 & 90 & 49 & 1
\end{tabular}

\begin{tabular}{l|l|l|l}
90 & 10 & 38 & 1
\end{tabular}

\begin{tabular}{l|l|l|l}
100 & 10 & 39 & 3
\end{tabular}

\begin{tabular}{l|l|l|l}
100 & 80 & 63 & 1
\end{tabular}

\begin{tabular}{l|l|l|l}
90 & 30 & 46 & 2
\end{tabular}

\begin{tabular}{l|l|l|l}
100 & 70 & 52 & 2
\end{tabular}

\begin{tabular}{l|l|l|l}
80 & 45 & 62 & 3
\end{tabular}

\begin{tabular}{l|l|l|l}
70 & 45 & 57 & 2
\end{tabular}

CTA; Cuff Tear Arthropathy, PHF; Proximal humerus fracture, HR; Hemiarthroplasty revision, *; Degree, F, Female, M; male

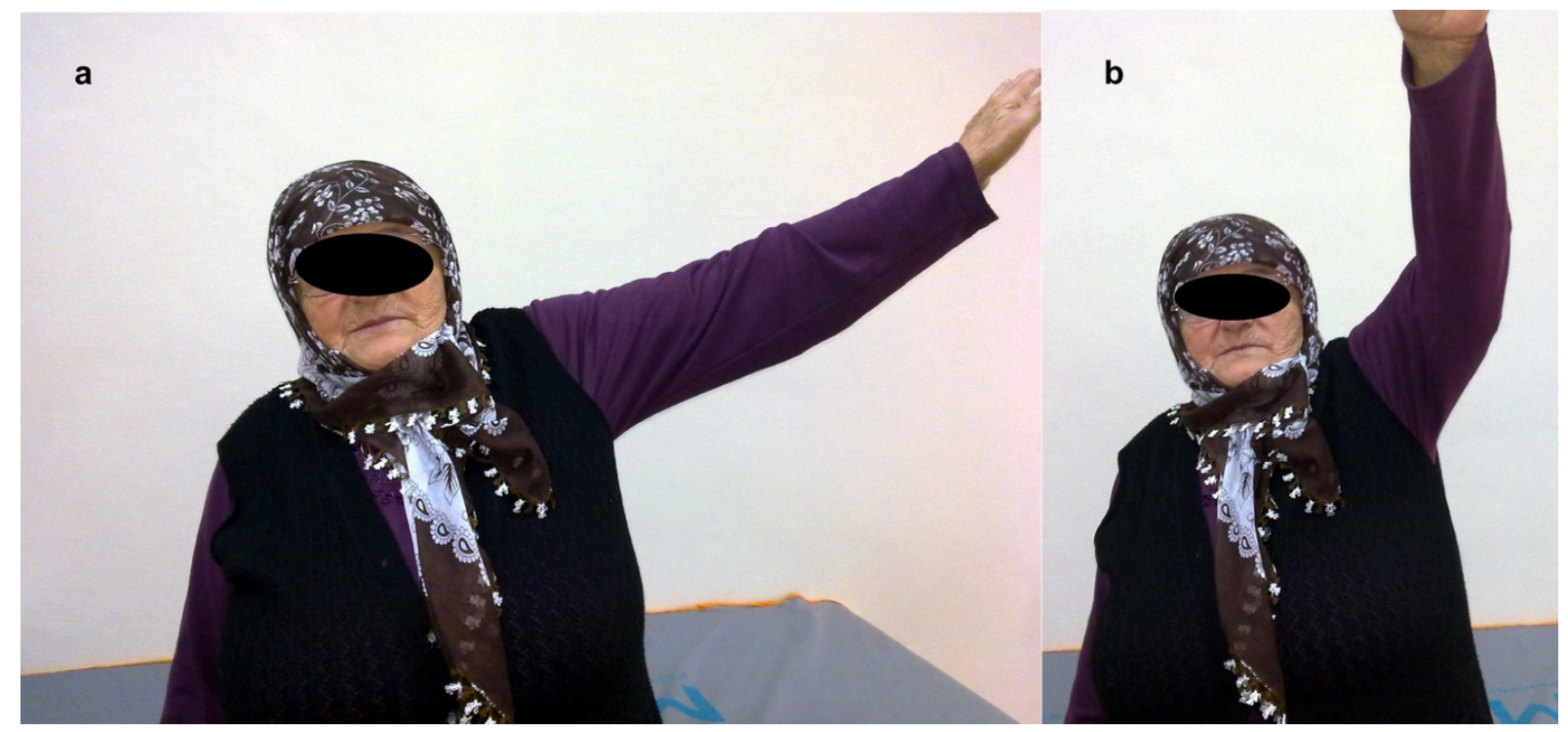

Fig. 2. Sixty-five-year-old patient with rotator cuff arthropathy and a reverse shoulder prosthesis, functional images of the $6^{\text {th }}$ month after surgery

Although the transacromial approach used by Grammont is less popular now, deltopectoral and anterosuperior approaches are the most used for RSA. Both approaches have advantages and disadvantages. The deltopectoral approach allows an easier approach to the glenoid lower pole and proximal humerus inferior, with the preservation of the deltoid muscle and active external rotation. With this approach, it helps to better position the glenoid component by reducing inferior compression and loosening. However, the disruption of the integrity of the subscapularis tendon is the risk of developing instability with wide capsular relaxation and the disability of the posterior cuff muscles and access to the glenoid (Ladermann et al., 2011).

The anterosuperior approach provides advantages such as simple and easy preparation of the humerus, easier approach to the glenoid, and preservation of the integrity of the subscapularis muscle. With this approach, the weakening of the anterior deltoid and the difficulty of the glenoid component placement are its disadvantages (Mole et al., 2011). The functional superiority of both approaches has not been reported (Ladermann et al., 2011). In all of our patients, we applied RSA with a deltopectoral approach.

Table 2. Comparison of patients' preoperative and final control data

\begin{tabular}{lllc} 
& \multicolumn{1}{c}{ Preoperative } & \multicolumn{1}{c}{ Last control } & \multicolumn{1}{c}{$\mathrm{P}^{*}$} \\
Active flexion $\left(^{\circ}\right)$ & $65(40-150)$ & $105(70-170)$ & $\geq 0.05$ \\
Active abduction $\left(^{\circ}\right)$ & $30(20-140)$ & $95(45-110)$ & $\geq 0.05$ \\
Internal rotation $\left(^{\circ}\right)$ & $40(20-90)$ & $30(10-90)$ & $\geq 0.05$ \\
External rotation $\left(^{\circ}\right)$ & $50(20-70)$ & $57.5(10-90)$ & $<0.05$ \\
Constant score & $20.5(14-63)$ & $54.5(38-64)$ & $<0.05$ \\
VAS & $7(3-9)$ & $1.5(1-3)$ & $<0.05$ \\
\hline
\end{tabular}

*Wilcoxon test 


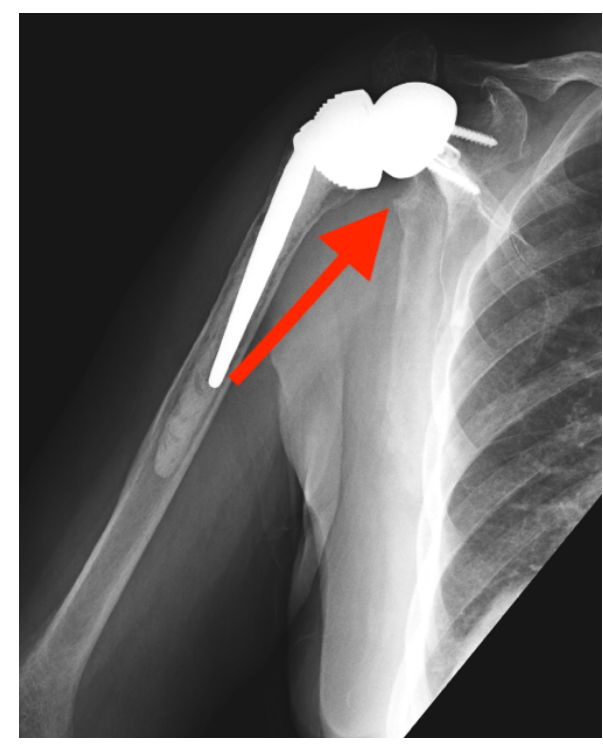

Fig. 3. 25-month control anteroposterior radiograph of a 78-year-old patient with an RSA for rotator cuff arthropathy. Scapular notching is indicated by the arrow

Complications of RSA have been reported as glenoid and humeral component loosening, dislocation, infection, scapular notching, periprosthetic fracture, neurological problems, acromion fracture, hematoma (Sirveaux et al., 2004; Werner et al., 2005; Boileau et al., 2006; Wall et al., 2007; Boileau et al., 2009; Cheung et al., 2011). Complication rates were higher in the RSA series performed as revision surgery (Wall et al., 2007). Dislocation has been reported as the most common complication (Cheung et al., 2011). In different studies, the incidence of scapular notching has been reported to be $44-96 \%$ (Vanhove and Beugnies, 2004; Werner et al., 2005; Simovitch et al., 2007; Wall et al., 2007; Levigne et al., 2008; Cazeneuve and Cristofari, 2010). Scapular notching was reported to be progressive and follow-up time and frequency were in direct proportion (Levigne et al., 2008). Different opinions have been reported on the clinical effect of scapular notching. Some authors report that notching does not affect shoulder range of motion or functional scores, but some report it as an unsuccessful result and independent risk factor for glenoid component loosening (Vanhove and Beugnies, 2004; Simovitch et al., 2007; Cazeneuve and Cristofari, 2010; Levigne et al., 2011). Cazeneuve and Cristofari (2010) observed a series of 36 patients with a RSA due to the proximal humerus fracture, with an average of 6.6 years follow-up in 23 patients with glenoid component loosening and 19 patients with scapular notching. Levigne et al. (2008) reported that notching was associated with preoperative rotator cuff arthropathy, but there was no relationship between the degree of notching and range of motion and Constant score. To prevent scapular notching, it is recommended to use eccentric gleno-sphere, to place the glenoid component downward and not to give superior tilt (Levigne et al., 2008; Levigne et al., 2011; Mizuno et al., 2012). However, there are authors who reported that placement of the glenoid component with the inferior tilt position does not decrease the rate of scapular notching (Edwards et al., 2012). The most effective methods of preventing inferior scapular notching were reported to use deltopectoral approach, the use of lateralized large-scale glenoid spheres and the insertion of components according to the patient's natural retroversion (Berhouet et al., 2014; Friedman et al., 2019). In patients in our study, we placed the glenoid sphere component inferiorly, preventing contact between the humeral component and the glenoid inferior. However, we found grade 1 scapular notching in one patient due to the superior tilt position of the glenoid. We did not find any complications in any of the patients except this patient.

The limitations of our study are that the patients with different diagnoses and the total number of patients are low and our follow-up time is short.

In conclusion, early results are good and complication rate is low in the treatment of RSA especially in patients with rotator cuff arthropathy. We recommend paying attention to the glenoid component location to avoid scapular notching. We continue our patients' follow-up to evaluate complications and functional results in the long term.

\section{Conflict of interest}

None to declare.

\section{References}

1. Baulot, E., Sirveaux, F., Boileau, P., 2011. Grammont's idea: The story of Paul Grammont's functional surgery concept and the development of the reverse principle. Clin. Orthop. Relat. Res. 469, 2425-2431.

2. Berhouet, J., Garaud, P., Favard, L., 2014. Evaluation of the role of glenosphere design and humeral component retroversion in avoiding scapular notching during reverse shoulder arthroplasty. J. Shoulder. Elbow. Surg. 23, 151-158.

3. Boileau, P., Watkinson, D., Hatzidakis, A.M., Hovorka, I., 2006. Neer Award 2005: The Grammont reverse shoulder prosthesis: results in cuff tear arthritis, fracture sequelae, and revision arthroplasty. J. Shoulder. Elbow. Surg. 15, 527-540.

4. Boileau, P., Gonzalez, J.F., Chuinard, C., Bicknell, R., Walch, G., 2009. Reverse total shoulder arthroplasty after failed rotator cuff surgery. J. Shoulder. Elbow. Surg. 18, 600-606.

5. Cazeneuve, J.F., Cristofari, D.J., 2010. The reverse shoulder prosthesis in the treatment of fractures of the proximal humerus in the elderly. J. Bone. Joint. Surg Br. 92, 535-539.

6. Cheung, E., Willis, M., Walker, M., Clark, R., Frankle, M.A., 2011. Complications in reverse total shoulder arthroplasty. J. Am. Acad. Orthop. Surg. 19, 439-449.

7. Edwards, T.B., Trappey, G.J., Riley, C., O'Connor, D.P., Elkousy, H.A., Gartsman, G.M., 2012. Inferior tilt of the glenoid component does not decrease scapular notching in reverse shoulder arthroplasty: results of a prospective randomized study. J. Shoulder. Elbow. Surg. 21, 641-646.

8. Friedman, R.J., Barcel, D.A., Eichinger, J.K., 2019. Scapular Notching in Reverse Total Shoulder Arthroplasty. J. Am. Acad. Orthop. Surg. 27, 200-209.

9. Grammont, P.M., Baulot, E., 1993. Delta shoulder prosthesis for rotator cuff rupture. Orthopedics. 16, 65-68.

10. Guery, J., Favard, L., Sirveaux, F., Oudet, D., Mole, D., Walch, G., 2006. Reverse total shoulder arthroplasty. Survivorship analysis of eighty replacements followed for five to ten years. J. 
Bone Joint Surg. Am. 88, 1742-1747.

11. Gunst, S., Louboutin, L., Swan, J., Lustig, S., Servien, E., NoveJosserand, L., 2021. Does healing of both greater and lesser tuberosities improve functional outcome after reverse shoulder arthroplasty for fracture? A retrospective study of twenty-eight cases with a computed tomography scan at a minimum of oneyear follow-up. International orthopaedics, 10.1007/s00264020-04928-9.

12. Ladermann, A., Lubbeke, A., Collin, P., Edwards, T.B., Sirveaux, F., Walch, G., 2011. Influence of surgical approach on functional outcome in reverse shoulder arthroplasty. Orthop. Traumatol. Surg. Res. 97, 579-582.

13. Levigne, C., Boileau, P., Favard, L., Garaud, P., Mole, D., Sirveaux, F., Walch, G., 2008. Scapular notching in reverse shoulder arthroplasty. J. Shoulder Elbow Surg. 17, 925-935.

14. Levigne, C., Garret, J., Boileau, P., Alami, G., Favard, L., Walch, G., 2011. Scapular notching in reverse shoulder arthroplasty: is it important to avoid it and how? Clin. Orthop. Relat. Res. 469, 2512-2520.

15. Levy, J.C., Badman, B., 2011. Reverse shoulder prosthesis for acute four-part fracture: tuberosity fixation using a horseshoe graft. J. Orthop. Trauma. 25, 318-324.

16. Matsen, F.A., Boileau, P., Walch, G., Gerber, C., Bicknell, R.T., 2007. The reverse total shoulder arthroplasty. J. Bone Joint Surg. Am. 89, 660-667.

17. Melis, B., Bonnevialle, N., Neyton, L., Levigne, C., Favard, L., Walch, G., Boileau, P., 2012. Glenoid loosening and failure in anatomical total shoulder arthroplasty: is revision with a reverse shoulder arthroplasty a reliable option? J. Shoulder Elbow Surg. 21, 342-349.

18. Mizuno, N., Denard, P.J., Raiss, P., Walch, G., 2012. The clinical and radiographical results of reverse total shoulder arthroplasty with eccentric glenosphere. Int. Orthop. 36, 16471653.

19. Mole, D., Wein, F., Dezaly, C., Valenti, P., Sirveaux, F., 2011. Surgical technique: the anterosuperior approach for reverse shoulder arthroplasty. Clin. Orthop. Relat. Res. 469, 2461-2468.
20. Ramirez, M.A., Ramirez, J., Murthi, A.M., 2012. Reverse total shoulder arthroplasty for irreparable rotator cuff tears and cuff tear arthropathy. Clin. Sports. Med. 31, 749-759.

21. Sebastia-Forcada, E., Lizaur-Utrilla, A., Mahiques-Segura, G., Ruiz-Lozano, M., Lopez-Prats, F. A., Alonso-Montero, C., 2020. Prospective comparison of functional outcomes of primary reverse shoulder arthroplasty for acute fractures versus rotator cuff deficiencies. The bone \& joint journal, 102, 15551559.

22. Shafritz, A.B., Flieger, S., 2012. Reverse total shoulder arthroplasty: early results of forty-one cases and a review of the literature. Hand Clin. 28, 469-79.

23. Simovitch, R.W., Zumstein, M.A., Lohri, E., Helmy, N., Gerber, C., 2007. Predictors of scapular notching in patients managed with the Delta III reverse total shoulder replacement. J. Bone Joint Surg. Am. 89, 588-600.

24. Sirveaux, F., Favard, L., Oudet, D., Huquet, D., Walch, G., Mole, D., 2004. Grammont inverted total shoulder arthroplasty in the treatment of glenohumeral osteoarthritis with massive rupture of the cuff. Results of a multicentre study of 80 shoulders. J. Bone Joint Surg. Br. 86, 388-395.

25. Schwarz, A. M., Hohenberger, G. M., Sauerschnig, M., Niks, M., Lipnik, G., Mattiassich, G., Zacherl, M., Seibert, F. J., Plecko, M., 2021. Effectiveness of reverse total shoulder arthroplasty for primary and secondary fracture care: mid-term outcomes in a single-centre experience. BMC Musculoskeletal Disorders, 22, 48.

26. Vanhove, B., Beugnies, A., 2004. Grammont's reverse shoulder prosthesis for rotator cuff arthropathy. A retrospective study of 32 cases. Acta Orthop. Belg. 70, 219-225.

27. Wall, B., Nove-Josserand, L., O'Connor, D.P., Edwards, T.B., Walch, G., 2007. Reverse total shoulder arthroplasty: A review of results according to etiology. J. Bone Joint Surg. Am. 89, $1476-1485$

28. Werner, C.M., Steinmann, P.A., Gilbart, M., Gerber, C., 2005. Treatment of painful pseudoparesis due to irreparable rotator cuff dysfunction with the Delta III reverse-ball-and-socket total shoulder prosthesis. J. Bone. Joint. Surg. Am. 87, 1476-1486. 